\title{
The effect of postoperative oral antibiotic therapy on the incidence of postoperative endophthalmitis after phacoemulsification surgery in dogs: 368 eyes (1997-2010)
}

\author{
Meg D. Sorhus ${ }^{1}$, Amanda Corr $^{1}$, Xiaocun Sun $^{2}$, Daniel A. Ward ${ }^{\text {Corresp. } 1}$ \\ ${ }^{1}$ Department of Small Animal Clinical Sciences, University of Tennessee College of Veterinary Medicine, Knoxville, Tennessee, United States of America \\ 2 Office of Information Technology, University of Tennessee, Knoxville, TN, USA \\ Corresponding Author: Daniel A. Ward \\ Email address: dward@utk.edu
}

Purpose. To assess the effectiveness of postoperative administration of oral antibiotics at reducing the incidence of endophthalmitis following phacoemulsification cataract extraction in dogs.

Methods. Medical records of the University of Tennessee College of Veterinary Medicine were reviewed for cases having undergone phacoemulsification and divided according to whether or not they had received oral antibiotics postoperatively. Records were then evaluated for a diagnosis of endophthalmitis and incidence rates between the group receiving postoperative oral antibiotics and the group not receiving postoperative oral antibiotics were compared.

Results. A total of 215 patients (368 eyes) were identified by the search. One-hundred twelve patients (197 eyes) were treated with oral antibiotics postoperatively. One-hundred and three patients (171 eyes) were not treated with oral antibiotics postoperatively. Three cases of endophthalmitis were identified, with one in the antibiotic-treated group and two in the non-antibiotic treated group ( $P>0.05$, Fisher's exact test).

Conclusions. The overall incidence of endophthalmitis at the University of Tennessee from 1997-2010 was $0.82 \%$. The rate of post-phacoemulsification endophthalmitis was unaffected by the postoperative administration of oral antibiotics. 
1 The effect of postoperative oral antibiotic therapy on the incidence of postoperative

2 endophthalmitis after phacoemulsification surgery in dogs: 368 eyes (1997-2010)

3 Meg D. Sorhus ${ }^{1}$, Amanda T. Corr ${ }^{1}$, Xiaocun Sun $^{2}$, Daniel A. Ward ${ }^{1}$

$4{ }^{1}$ Department of Small Animal Clinical Sciences, University of Tennessee College of Veterinary

5 Medicine, Knoxville, TN, 37996 USA

$6 \quad{ }^{2}$ Office of Information Technology, University of Tennessee, Knoxville, TN, 37996 USA

7 Corresponding author: Daniel A. Ward ${ }^{1}$

8 


\section{Abstract}

10 Purpose. To assess the effectiveness of postoperative administration of oral antibiotics at

11 reducing the incidence of endophthalmitis following phacoemulsification cataract extraction in

12 dogs.

13 Methods. Medical records of the University of Tennessee College of Veterinary Medicine were

14 reviewed for cases having undergone phacoemulsification and divided according to whether or

15 not they had received oral antibiotics postoperatively. Records were then evaluated for a

16 diagnosis of endophthalmitis and incidence rates between the group receiving postoperative oral

17 antibiotics and the group not receiving postoperative oral antibiotics were compared.

18 Results. A total of 215 patients (368 eyes) were identified by the search. One-hundred twelve

19 patients (197 eyes) were treated with oral antibiotics postoperatively. One-hundred and three

20 patients (171 eyes) were not treated with oral antibiotics postoperatively. Three cases of

21 endophthalmitis were identified, with one in the antibiotic-treated group and two in the non-

22 antibiotic treated group $(\mathrm{P}>0.05$, Fisher's exact test $)$.

23 Conclusions. The overall incidence of endophthalmitis at the University of Tennessee from

$24 \quad 1997-2010$ was $0.82 \%$. The rate of post-phacoemulsification endophthalmitis was unaffected by

25 the postoperative administration of oral antibiotics. 


\section{Introduction}

28 Infectious endophthalmitis is one of the most devastating complications of phacoemulsification cataract extraction in both human and veterinary ophthalmology. In humans, postphacoemulsification endophthalmitis results in visual acuity of $20 / 200$ or worse in $15-30 \%$ of cases, and 10\% are left with no useful vision (20/800 or less) (Behndig et al, 2013; Durand 2013). The rate of post-cataract extraction endophthalmitis ranges from $0.012 \%$ to $0.56 \%$ in the human literature (Liesegang, 2001; Ciulla, Starr \& Masket, 2002; Kamalarajah et al, 2004; Li et al, 2004; Taban et al, 2005; Wejde et al, 2005; Ou \& Ta, 2006; Rosha et al, 2006; Cao et al, 2013; Rudnisky, Wan \& Weis, 2014), with two large meta-analyses reporting average rates of $0.134 \%(n=6,686,169$; Cao et al, 2013) and 0.128\% $(n=3,140,560$; Taban et al, 2005). Rates from 0 - 1.4\% have been published in dogs (Sigle \& Nasisse, 2006; Johnstone \& Ward, 2005; Azoulay et al, 2013; Ledbetter, Spertus \& Kurtzman, 2018; Lacerda et al, 2018). The presumed sources of ocular infection in humans are the eyelids and conjunctival surface (Speaker et al, 1991) and risk factors include advanced age, immunosuppressive comorbidities (e.g., diabetes mellitus), and intraoperative complications (Sengillo et al, 2020); the same are presumed to be true for veterinary patients (Ledbetter et al, 2018). Although variations do exist, standards of care for prevention of infection during cataract surgery among human medical institutions have been suggested ( Rosha et al, 2006; Rudnisky, Wan \& Weis, 2014; Behndig et al, 2013). Many veterinary practices likely have been extrapolated from this data. However, there is still much opportunity to investigate the best practices for prevention of endophthalmitis in veterinary patients undergoing cataract surgery.

While intracameral antibiotic administration has found widespread acceptance as

bacterial endophthalmitis prophylaxis (Haripriya et al, 2017), the most widely cited strategy, and 
50 the approach with the highest level of evidence of efficacy, is the preoperative use of $5 \%$

51 povidone-iodine on the periocular and ocular surfaces (Speaker \& Menikoff, 1991; Taylor et al,

52 1995; Liesegang, 2001; Ciulla, Starr \& Masket, 2002; Mayer et al, 2003; Ou \& Ta, 2006; Rosha

53 et al, 2006; Sengillo et al, 2020). Perioperative administration of topical antibiotics is common

54 in both human and veterinary cataract surgery, despite a lack of evidence that they reduce the

55 incidence of post-operative bacterial endophthalmitis (Grzybowski et al, 2016; Endophthalmitis

56 Study Group, 2007; Huang et al, 2016).

57 Although there is no evidence that postoperative systemic antibiotic administration

58 reduces the incidence of postoperative bacterial endophthalmitis, many veterinary

59 ophthalmologists list postoperative systemic antibiotic administration as part of their

60 perioperative protocols (Paulsen et al, 1986; Boldy, 1988; Taylor et al, 1995; Ledbetter,

61 Millichamp \& Dziezyc, 2004; Sigle \& Nasisse, 2006; Hazra et al, 2008; Gift et al, 2009;

62 Ledbetter, Spertus \& Kurtzman, 2018). Postoperative oral antibiotics are not used routinely

63 following cataract extraction in humans; in one study of endophthalmitis prophylaxis practices,

64 postoperative oral antibiotics were not used in any of the 75,318 cataract extractions that were

65 reviewed (Rudnisky, Wan \& Weis, 2014). Prophylactic use of postoperative systemic antibiotics

66 is particularly problematic due to the potential for antibiotic resistance, adverse side effects,

67 additional cost and additional medication for owners to administer. Up to $50-60 \%$ of operations

68 in human general surgery are estimated to be associated with over-use, under-use or misuse of

69 antibiotics (Bratzler et al, 2005; De Almeida et al, 2018). Recent guidelines made by the

70 American Journal of Veterinary Internal Medicine have been made in response to recognition of

71 the importance of veterinarians' role in reducing antimicrobial resistance whenever possible

72 (Weese et al, 2015). 
The purpose of this retrospective study was to determine whether prolonged post-

operative oral antibiotic administration is effective in reducing the incidence of endophthalmitis

after phacoemulsification in dogs, and we hypothesized that a statistically significant effect

would not be found.

Materials and Methods

Medical records of patients that had phacoemulsification performed at the University of

Tennessee Veterinary Teaching Hospital (UTVTH) between 1/1/1997 and 12/31/2010 were

reviewed for procedure codes of "cataract surgery" or "phacoemulsification" and a diagnosis code of "endophthalmitis." This period of time was evaluated because in that timeframe some of the cataract patients at UTVTH received oral antibiotics following cataract extraction while others did not. Cases receiving pre- and/or post-operative topical antibiotics other than neomycin-polymixin B-gramicidin, cases with evidence of post-operative corneal ulceration and patients that did not return for at least three post-operative follow-up appointments were excluded from the study. Records were evaluated for breed, patient age, type of intraocular lens (IOL) that was implanted, level of experience of surgeon (i.e., diplomate of the American College of Veterinary Ophthalmologists (ACVO) vs. ophthalmology resident), diabetic status, postoperative ocular hypertension (defined as intraocular pressure (IOP) $>25 \mathrm{mmHg}$ within 24 hours of surgery) and operative time. ophthalmoscopy, Schirmer tear testing, fluorescein staining, applanation tonometry, $15 \mathrm{MHz}$ ocular ultrasonography, and flash electroretinography (ERG). Unless otherwise specified, ultrasonography and electroretinography were not performed on recheck examinations. Urine 
96 were treated prior to surgery. Examinations were performed by an ACVO diplomate, a resident

97 in an ACVO-approved residency program, or both. Surgeries were performed by an ACVO

98 diplomate, a third year veterinary ophthalmology resident, or a first or second year veterinary

99 ophthalmology resident under the direct supervision of an ACVO diplomate. A total of eight

100 different surgeons performed surgeries included in the timeframe of this study (two ACVO dipl.,

101 six residents). Surgery was standard one- or two-handed phacoemulsification, per surgeon's

102 preference, and instruments were not changed between eyes of bilateral surgeries. In general,

103 pre-operative protocols consisted of treatment with topical anti-inflammatory medication

104 (steroidal and/or nonsteroidal) BID for approximately 7 days prior to surgery. With slight

105 variation in timing, treatment protocols for the day prior to and morning of surgery consisted of

106 topical 1\% prednisolone acetate QID, topical 0.03\% flurbiprofen QID, topical 0.175\%

107 neomycin-0.0025\% gramicidin-10,000 units/mL polymixin B QID, topical $2.5 \%$ phenylephrine

108 QID, and systemic flunixin meglumine $(0.44 \mathrm{mg} / \mathrm{kg} \mathrm{IV} \mathrm{immediately} \mathrm{prior} \mathrm{to} \mathrm{anesthetic}$

109 induction) or carprofen (2.2 mg/kg SQ 2 hours prior to anesthetic induction). Cefazolin

$110(22 \mathrm{mg} / \mathrm{kg} \mathrm{IV})$ was administered pre- and intra-operatively beginning at anesthetic induction and

111 continued every 90 minutes until the end of surgery. Polymethylmethacrylate (PMMA) or

112 foldable acrylic intraocular lenses (IOLs) were inserted within the lens capsule when appropriate

113 at the discretion of the surgeon. The types of PMMA and acrylic IOLs used did not change over

114 the study period. Post-operative protocols generally consisted of topical anti-inflammatories,

115 antibiotics, parasympatholytics, and artificial tears. In addition, some patients received

116 subconjunctival corticosteroids (0.5-1.0 mg dexamethasone sodium phosphate) or systemic

117 corticosteroids (prednisolone $0.5 \mathrm{mg} / \mathrm{kg}$ PO QD-BID for 5-10 days). No specific reasoning for

118 opting to administer subconjunctival corticosteroids was discernable from the medical records. 
119 Systemic corticosteroids were given in cases of posterior capsule disruption or severe uveitis the

120 day following surgery.

121 Oral post-operative antibiotics were used in some cases but not in others based upon

122 surgeon's preference and training; review of the medical records did not identify any patient,

123 surgical, environmental, client, or other variables that might have influenced this decision.

124 When prescribed, oral antibiotics were usually either amoxicillin $(n=34)$, cefadroxil $(n=62)$ or

125 cephalexin $(\mathrm{n}=13)$ dosed at 20-30 mg/kg PO BID for 7-10 days, based upon surgeon's

126 preference, availability at our pharmacy, and cost. Three dogs were on different antibiotics at

127 surgical admission for non-ocular reasons. One was on cefpodoxime $11 \mathrm{mg} / \mathrm{kg}$ PO QD for 30

128 days to treat pyoderma, one was on enrofloxacin $5 \mathrm{mg} / \mathrm{kg}$ PO BID for seven days to treat a

129 prostatic abscess, and one was on amoxicillin/clavulanic acid $13 \mathrm{mg} / \mathrm{kg}$ PO BID for 14 days.

130 The oral antibiotic therapy for these dogs was not changed following cataract surgery.

$131 \quad$ All operated patients were rechecked 1 day postoperatively. Additional routine recheck

132 examinations were planned for approximately 7 days, 21 days, and 56 days postoperatively;

133 actual ranges of these rechecks were 4-11 days, 20-29 days, and 36-78 days respectively for the

134 antibiotic-treated group, and 5-10 days, 19-27 days, and 40-57 days for the non-antibiotic-treated

135 group. Additional recheck exams were conducted if deemed necessary by the attending clinician

136 or requested by the owner. Patients that did not return to UTCVM for at least the first three

137 scheduled rechecks were not included in the study. Post-operative endophthalmitis was

138 diagnosed by an ACVO diplomate based upon clinical examination finding of severe anterior

139 uveitis (characterized by $3+/ 4+$ or $4+/ 4+$ anterior chamber flare and cells) with hypopyon,

140 decreased vision (as indicated by loss of menace response), and ocular pain beyond what would

141 be expected post-operatively, with or without a positive aqueous humor culture, as per Rudnisky 
142 et al (Rudnisky, Wan \& Weis, 2014). Histopathology of one enucleated eye was performed by a

143 diplomate of the American College of Veterinary Pathologists (ACVP) or a resident in an

144 ACVP-approved residency.

145 Each eye that underwent phacoemulsification was considered the experimental unit for

146 the purpose of statistical analysis. Descriptive statistics were reported as median together with

147 either range or $25^{\text {th }}$ and $75^{\text {th }}$ percentiles. Chi square (or 2-tailed Fisher's exact test when

148 contingency table values $<5$ ) was used to compare categorical data (including the rate of

149 endophthalmitis in dogs that did receive postoperative systemic antibiotics with those that did

150 not) using commercially available software ${ }^{a}$. The Mann-Whitney U test was used to compare

151 continuous data between the 2 groups. $\mathrm{P}<0.05$ was considered statistically significant.

\section{Results}

153 A total of 215 patients and 368 eyes were identified by the medical records search as

154 satisfying inclusion and exclusion criteria. Median follow-up times were 412.5 days in the

155 antibiotic-treated group (range 48-1800 days) and 220 days (range 24-696 days) in the non-

156 antibiotic treated group. The distribution of breeds and IOL types were similar between the two

157 groups (see Table 1). Similarly, there were no differences between the two groups with respect

158 to age, proportion of eyes operated by ACVO diplomates vs. residents, proportion of diabetic

159 patients, proportion of eyes with IOP $>25 \mathrm{mmHg}$ within 24 hours of surgery, or operative time 160 (see Table 1).

161 One-hundred and twelve patients were treated and 103 patients were not treated with

162 systemic oral antibiotics post-operatively. Among the 112 antibiotic-treated patients, 85 were

163 operated bilaterally and 27 were operated unilaterally for a total of 197 eyes in the antibiotic-

164 treated group. Among the 103 patients not treated with postoperative oral antibiotics, 68 were 
165 operated bilaterally and 35 were operated unilaterally for a total of 171 eyes in this group.

166 During the study period, three eyes of three different patients (one eye from the antibiotic-treated

167 group and two from the non-antibiotic-treated group) developed post-operative endophthalmitis,

168 for an overall incidence of $3 / 368$, or $0.82 \%$. There was no statistically significantly difference in

169 incidence rates between the two groups $(\mathrm{p}=0.599$; power at $\alpha=0.05: 0.370$ to detect a 5 -fold

170 difference, 0.763 to detect a 10 -fold difference).

171 Endophthalmitis Cases

172 The first affected patient (from the antibiotic-treated group) was a 12 year old

173 nondiabetic female spayed mixed breed dog with bilateral immature cataracts. Apart from the

174 cataracts, ocular examination was within normal limits. Preoperative ERG was normal with a-

175 wave:b-wave amplitude $>100 \mu \mathrm{V}$ in both eyes (OU), and ocular ultrasonography showed mature

176 cataracts with no evidence of retinal detachment, lens capsule rupture or vitreal degeneration

177 bilaterally. The dog underwent uneventful bilateral phacoemulsification. This patient was left

178 aphakic OU; the reason for that decision was not elucidated in the medical record, but the

179 surgery report did not indicate lens instability nor posterior capsular tears. The surgical time in

180 this patient was 40 minutes in each eye. Immediate postoperative treatment consisted of a single

181 subconjunctival injection of $0.4 \mathrm{mg}$ dexamethasone sodium phosphate, prednisolone acetate (1

182 drop OU q 4 hours), neomycin-polymixin B-gramicidin (1 drop OU q 8 hrs), prednisone (1

$183 \mathrm{mg} / \mathrm{kg}$ PO q $12 \mathrm{hrs}$ ) and cephalexin (25 mg/kg PO q $8 \mathrm{hrs})$. On postoperative day (POD) 1 an

184 incisional wound leak was present and cyanoacrylate tissue adhesive was applied. The incision

185 was still leaking on POD 2, so the patient was taken back to surgery and the sutures were

186 removed and replaced without complication. The dog was discharged on POD 3 on a regimen of

187 prednisolone acetate (1 drop OU q 6 hours), neomycin-polymixin B-gramicidin (1 drop OU q 8 
$188 \mathrm{hrs}$ ), tropicamide (1 drop OU q $12 \mathrm{hrs}$ ), oral prednisone at a tapering dose ( $1 \mathrm{mg} / \mathrm{kg}$ PO q $12 \mathrm{hrs}$

$189 \mathrm{X} 4$ days, then q $24 \mathrm{hrs}$ ), and cephalexin (25 mg/kg PO q 8 hrs for 10 days). On POD 15, recheck

190 examination demonstrated that there was no aqueous flare OU, no wound leaks OU, no corneal

191 ulcers OU, and IOPs of $26 \mathrm{mmHg}$ in the right eye (OD) and $23 \mathrm{mmHg}$ in the left eye (OS).

192 Vision was considered good based on positive menace responses OU and ability to successfully

193 navigate an obstacle course in the exam room. Prednisolone acetate was decreased to 1 drop

194 OU q 8 hrs, neomycin-polymixin B-gramicidin was continued at 1 drop OU q 8 hrs, and

195 tropicamide was decreased to q $24 \mathrm{hrs}$. Oral prednisone was decreased to $1 \mathrm{mg} / \mathrm{kg} \mathrm{PO} \mathrm{q} 48 \mathrm{hrs}$

196 for another week and then discontinued.

197 On POD 19, the patient returned after the owner noticed acute onset redness, cloudiness,

198 pain, and periocular swelling OS. Exam OD showed no aqueous flare, no wound leaks, no

199 corneal ulcers, and an IOP of $20 \mathrm{mmHg}$. The OS had conjunctival and episcleral injection,

200 aqueous leakage at the incision site, 3+ diffuse corneal edema, 4+ aqueous flare and cells,

201 hypopyon, fibrin in the anterior chamber and miosis OS. The dog was judged to be blind OS

202 based on a negative menace response. The IOP was $30 \mathrm{mmHg}$ OS. The presumptive diagnosis

203 was endophthalmitis and secondary glaucoma. Aerobic, anaerobic and fungal cultures were

204 obtained from the wound leak and from an aqueous humor paracentesis. Both sites yielded

205 positive growth of Enterococcus faecalis that was sensitive to all antibiotics tested (ampicillin,

206 chloramphenicol, penicillin, vancomycin, gentamicin and streptomycin); anaerobic and fungal

207 cultures were negative. An ultrasound OS identified a complete retinal detachment with cellular

208 infiltrate in the vitreal chamber. Due to the ocular pain and poor prognosis for vision, the left

209 eye was enucleated. The eye was submitted for histopathologic examination, which revealed

210 corneal edema, large numbers of neutrophils admixed with fibrin in the anterior and posterior 
211 chambers, neutrophilic infiltration of the iris, ciliary body and choroid, retinal detachment,

212 degeneration of the photoreceptor layer, and retinal pigment epithelial hypertrophy. No

213 microorganisms were identified on histopathology.

214 The second affected patient (from the non-antibiotic-treated group) was a 12 year old

215 diabetic male castrated mixed breed dog with mature cataracts OU. In addition to the cataracts,

216 lens instability due to zonular disruption was noted OD. Therefore, an intracapsular extraction

217 was performed OD and an IOL was not placed. Surgery OS was uneventful and a PMMA IOL

218 was placed in that eye. Operative times were 45 mins OD, 85 mins OS. Post-operatively, the

219 patient was started on prednisolone acetate (1 drop OU q 4 hours), neomycin-polymixin B-

220 gramicidin (1 drop OU q 4 hrs), and ocular lubricating gel (1/4” OU q 4 hrs). The dog was

221 discharged on POD 2 on a regimen of prednisolone acetate (1 drop OU q 6 hours), neomycin-

222 polymixin B-gramicidin (1 drop OU q $6 \mathrm{hrs}$ ), tropicamide (1 drop OU q $8 \mathrm{hrs}$ ), and ocular

223 lubricating gel (1/4" OU q 4 hrs). Systemic antibiotics were not prescribed. Recheck exams on

224 PODs 9 and 23 were normal (no flare OU, normal IOPs OU, no ulcers OU) with the exception of

225 retinal hemorrhage OS, which was attributed to diabetic retinopathy (Landry, Herring \&

226 Panciera, 2004). The tropicamide and neomycin-polymixin B-gramicidin were discontinued and

227 the prednisolone acetate was decreased to $\mathrm{q} 24 \mathrm{hrs}$.

228 On POD 30, the patient was re-presented for a scheduled recheck and had 3+ aqueous

229 flare and cells and hypopyon OS. The IOP was $5 \mathrm{mmHg}$. The patient was diagnosed with

230 anterior uveitis and presumptive endophthalmitis OS. The patient was discharged on

231 enrofloxacin (5 mg/kg PO BID), prednisolone acetate (1 drop up to QID OS) and atropine (1

232 drop OS TID). On POD 35 flare and hypopyon were reduced, though fibrin and hyphema were

233 present. The IOP OS was too low to read. All medications were continued. The 
234 endophthalmitis OS continued to show subjective improvement and on POD 44 the enrofloxacin

235 was discontinued while the prednisolone acetate and atropine were continued as previously

236 prescribed. By POD 65 the endophthalmitis was considered resolved with no hyphema, fibrin,

237 flare or hypopyon present. The prednisolone acetate was decreased (TID X 2 weeks and then

238 BID X 2 weeks) and the atropine was decreased (BID). All medications were discontinued on

239 POD 86, at which time menace responses were positive OU and functional vision was deemed to 240 be excellent. The patient was lost to follow-up after this visit.

241 The third affected patient (also from the non-antibiotic-treated group) was an 11 year old

242 nondiabetic male castrated toy poodle with a late immature cataracts OD and hypermature

243 cataract OS. Medical history included recurrent pyodermas, but the dog was otherwise in good

244 health. Intraoperatively significant zonular disruption was noted OS, so following lens removal

245 the lens capsule was also removed and no IOL was placed in that eye. Surgery OD was

246 uneventful and a PMMA IOL was placed in that eye. Operative times were 60 mins OS and 65

247 mins OD. Post-operatively, the patient was started on prednisolone acetate (1 drop OU q 4

248 hours), neomycin-polymixin B-gramicidin (1 drop OU q 4 hrs), and ocular lubricating gel (1/4"

249 OU q 4 hrs). Operative times were 45 mins OD and 85 mins OS. The evening of surgery the

250 IOP OS rose to $26 \mathrm{mmHg}$, so the dog was placed on dorzolamide/timolol 1 drop OS BID. On

251 POD 1 the IOP had dropped to $9 \mathrm{mmHg}$ OS, and the dog was discharged on a regimen of

252 prednisolone acetate (1 drop OU q 6 hours), neomycin-polymixin B-gramicidin (1 drop OU q 6

253 hrs), tropicamide (1 drop OU q 8 hrs), ocular lubricating gel (1/4” OU q 4 hrs) and

254 dorzolamide/timolol ( 1 drop OS q 12 hrs). Systemic antibiotics were not prescribed. Recheck

255 examinations on PODs 8, 21, 44 and 56 were unremarkable apart from a mild IOP increase OS 
256

257

258

259

260

261

262

263

264

265

266

267

268

269

270

271

272

273

274

275

276

277

278

on POD 21 (25 mmHg). By POD 44 the IOP OS had returned to normal at $6 \mathrm{mmHg}$. Between PODs 8 and 56 were gradually weaned and discontinued.

On POD 87 the dog was presented for acute onset blepharospasm and discharge OS of 23 days' duration. Ocular exam revealed hypopyon completely filling the anterior chamber so that the iris was only visible at it's periphery. The eye was diagnosed with endophthalmitis and treated with topical ciprofloxacin (1 drop OS q $2 \mathrm{hrs}$ ), and oral enrofloxacin ( $5 \mathrm{mg} / \mathrm{kg}$ PO BID) and amoxicillin/clavulanic acid (13 mg/kg PO BID). The dog was rechecked on POD 89, and no improvement was noted. An ocular ultrasound revealed hyperechoic material consistent with purulence and/or blood in the anterior chamber and vitreous cavity, with no obvious retinal detachment. We recommended an anterior chamber tap for cytology and culture, but the owner declined and opted to have the eye enucleated at the referring veterinarian's office. We requested that the referring veterinarian obtain an intraocular culture at the time of enucleation and to have the eye returned to us for histopathology, but neither of these requests were honored.

\section{Discussion}

Our data indicate that endophthalmitis following phacoemulsification cataract extraction is a rare event in dogs, occurring in 3 out of 368 at risk eyes in this study $(0.82 \%)$. This is in general agreement with previous studies in dogs (Johnstone \& Ward, 2005; Sigle \& Nasisse, 2006; Azoulay et al, 2013; Ledbetter, Spertus \& Kurtzman, 2018) but higher than most studies in humans (Liesegang, 2001; Ciulla, Starr \& Masket, 2002; Kamalarajah et al, 2004; Li et al, 2004;

Taban et al, 2005; Wejde et al, 2005; Ou \& Ta, 2006; Rosha et al, 2006; Cao et al, 2013;

Rudnisky, Wan \& Weis, 2014). Furthermore, our data did not reveal that postoperative administration of systemic antibiotics protected against endophthalmitis. With the diagnosis of endophthalmitis being so rare in both groups, one must be wary of statistical serendipity hiding 
279 significant differences. The power to detect treatment effects of 5-fold and 10 -fold were 0.360

280 and 0.763 , respectively. Our findings would be more convincing had the power exceeded 0.80 ,

281 which would have required an increase in sample size. However, it can be calculated that an

282 additional 6 cases would have had to occur in the group not receiving systemic antibiotics (with

283 no additional cases in the group that did receive systemic antibiotics) to demonstrate a

284 statistically significant protective effect of systemic antibiotics. Given that we only found 2

285 cases in the untreated cohort of 171 eyes, it is highly unlikely that our conclusions would change

286 if the study were extrapolated to thousands of cases.

287 The cases in this report developed more than a week postoperatively, analogous to what

288 some authors in the human literature designate "chronic post-phacoemulsification

289 endophthalmitis" (Durand, 2013); other authors describe cases occurring within 6 weeks of

290 surgery as "acute" and those occurring more than 6 weeks after surgery as "delayed onset"

291 (Miller et al, 2005) Most cases of endophthalmitis following phacoemulsification probably

292 occur following introduction of infectious agents from the conjunctiva and eyelid margins

293 (Sherwood et al 1989; Tervo et al, 1999; Liesegang, 2001; Ledbetter, Millichamp \& Dziezyc,

294 2004), although Lacerda et al demonstrated that sources of contamination other than the ocular

295 surface do occur in canine phacoemulsification (Lacerda et al, 2018). The third patient affected

296 with endophthalmitis in this report deserves particular attention. The diagnosis in that case was

297 made on POD 87, which is an extraordinarily long time even for chronic or delayed-onset post-

298 phacoemulsification endophthalmitis (Durand 2013), and we question whether that case truly

299 represents an infection related to surgery. We included that case simply because it did fit our

300 predetermined inclusion criteria; if one were to eliminate that case it would only strengthen our

301 conclusion that postoperative antibiotics do not reduce the incidence of postoperative 
302 endophthalmitis as that case was in the group that did not receive antibiotics after surgery. If that

303 case were eliminated, the total endophthalmitis incidence in our study would be $2 / 366(0.55 \%)$,

304 with $1 / 197(0.51 \%)$ in the antibiotic-treated group and 1/169 $(0.59 \%)$ in the non-antibiotic-

305 treated group.

306 In humans the most common offending organisms are coagulase negative Staphylococci

307 in acute cases (i.e., POD 2-7) and Propionibacterium acnes in more chronic cases (Durand,

308 2013). The single culture-proven case in the present study was an Enterococcus, but the number

309 of reported cases of post-phacoemulsification endophthalmitis in dogs is too limited to make any

310 conclusions about predominance of any specific organism (Johnstone \& Ward, 2005; Sigle \&

311 Nasisse, 2006; Ledbetter, Spertus \& Kurtzman, 2018). It has been shown that introduction of

312 organisms into the eye during phacoemulsification is rather common in dogs and humans, yet

313 development of endophthalmitis is rare (Dickey, Thompson \& Jay, 1991; Taylor et al, 1995;

314 Tervo et al, 1999; Ledbetter, Millichamp \& Dziezyc, 2004; Ledbetter, Spertus \& Kurtzman,

315 2018). This indicates that the eye has effective mechanisms for clearing organisms from the

316 anterior chamber prior to the development of infection, with the most important mechanism

317 being constant turnover of aqueous humor (Durand, 2013).

318 Reported risk factors for developing post-phacoemulsification endophthalmitis have been

319 identified in humans and include posterior capsule disruption, incisional wound leak, concurrent

320 diabetes mellitus, concurrent periocular disease, immunoincompetence, and advanced age

321 (Aaberg et al, 1998; Liesegang, 1999; Liesegang, 2001; Li et al, 2004; Wejde et al, 2005; Ou \&

322 Ta, 2006; Cao et al, 2013; Rahmani \& Eliot, 2018). It is interesting to note that all of our

323 endophthalmitis cases each had one of these risk factors. Case 1 had a wound leak, case 2 was a

324 diabetic, and case 3 had posterior capsule removal due to zonular instability, also necessitating 
325 anterior vitrectomy. Other factors reported to influence postoperative endophthalmitis rates in

326 humans include surgical technique (with manual extracapsular extraction associated with higher

327 rates than phacoemulsification), corneal incision type (with clear corneal incisions associated

328 with higher rates than scleral tunnel incisions), and choice of intraocular lens (with injectable

329 lenses associated with lower rates than non-injectable lenses) (Mayer et al, 2003; Miller et al,

330 2005; Ou \& Ta, 2006; Rosha et al, 2006; Endophthalmitis Study Group, 2007).

331 Among methods of reducing the incidence of post-phacoemulsification endophthalmitis,

332 surgical preparation with $5 \%$ povidone-iodine is most consistently supported in the literature

333 (Speaker \& Menikoff, 1991; Liesegang, 1999; Schmitz et al, 1999; Liesegang, 2001; Ciulla,

334 Starr \& Masket, 2002; Ang \& Barras, 2006; Ou \& Ta, 2006). There is no evidence that

335 prolonged postoperative systemic antibiotics are beneficial in reducing the incidence of

336 postoperative endophthalmitis in humans (Liesegang, 2001; Ang \& Barras, 2006), and our data

337 suggest the same is true in dogs. The numerous untoward effects of injudicious use of antibiotics

338 makes it clear that without evidence of active infection systemic antibiotics should not be

339 administered after phacoemulsification in dogs. For preoperative antimicrobial prophylaxis in

340 general surgery, the Surgical Infection Prevention Guideline Writers Workgroup advocates

341 administering a first intravenous antimicrobial dose within 60 minutes before surgical incision

342 and discontinuing antimicrobials no more than 24 hours after the end of surgery (Bratzler \&

343 Houck, 2005). It seems prudent to follow these recommendations for phacoemulsification in

344 dogs.

Preoperative topical antibiotics are also widely used as endophthalmitis prophylaxis, and

346 while reports of efficacy are conflicting most cataract surgeons continue to use them (Lehmann

347 et al, 1997; Liesegang, 1999; Ta et al, 2002; de Kaspar et al, 2004; Ou \& Ta, 2006; Huang et al, 
348 2016). In one review of perioperative practices aimed at preventing postoperative

349 endophthalmitis, $76.5 \%$ of cases received preoperative topical antibiotics, but the specific agents

350 utilized were not specified (Inoue et al, 2018). Another meta-analysis reported wide variability

351 in specific agents used, including fluoroquinolones, aminoglycosides, cephalosporins, and

352 chloramphenicol (Haripriya et al, 2017). Some studies have also shown a protective effect of

353 subconjunctival antibiotics and intracameral antibiotics (Schmitz et al, 1999; Rosha et al, 2006;

354 Endophthalmitis Study Group, 2007), while other studies have found no benefit from these

355 measures (Ciulla, Starr \& Masket, 2002). The preponderance of the evidence from the most

356 recent evaluations of intracameral antibiotic use following phacoemulsification in humans

357 strongly suggest that they do have a protective effect against endophthalmitis, with the major

358 limitations being availability of commercially available products approved for that use and

359 complications associated with compounded products (Haripriya \& Chang, 2018).

360 In some particular respects our data must be viewed with caution. Conclusions drawn in

361 retrospective studies are only as reliable as the medical record entries that give rise to them; this

362 variable cannot be controlled after the fact. As with all retrospectives, subjects could not be

363 randomized into oral antibiotic-treated and non-antibiotic-treated groups. It would also have

364 been optimal had all dogs in the antibiotic-treated group received the same antibiotic. However,

365 given that 109 of 112 dogs in this group received $\beta$-lactam antibiotics with similar spectra (as

366 were 2 of the other 3 dogs who were already on oral antibiotics when admitted for surgery) it is

367 unlikely that results would have been different had oral antibiotic administration been more

368 consistent. Additionally, while 368 operated eyes would appear to be a relatively large sample

369 size, it is suboptimal when studying a condition of extreme rarity; similar studies in the human

370 literature usually include 10,000 - 100,000 subjects, and meta-analyses may include millions 
371 (Aaberg et al, 1998; Mayer et al, 2003; Li et al, 2004; Wejde et al, 2005; Cao et al, 2013). It

372 would also be more convincing if all suspected cases had positive cultures, but it has been

373 established in the human endophthalmitis literature that many cases proven to be associated with

374 bacterial infection on PCR testing are negative on microbiological culture (Therese, Anand \&

375 Madhavan, 1998; Kosacki et al, 2020). Thus, bacterial endophthalmitis following

376 phacoemulsification is often a clinical diagnosis based on ocular signs and time to occurrence

377 (Durand, 2013; Rudnisky, Wan \& Weis, 2014), and Kamalarajah et al even refer to the

378 syndrome as "presumed infectious endophthalmitis" (Kamalarajah et al, 2004). Nevertheless, it

379 should be considered the standard of practice that vitreal cultures and microbial PCR testing be

380 performed on suspected postoperative endophthalmitis cases. It has been speculated that sterile

381 endophthalmitis can occur following cataract extraction (Ledbetter et al, 2018), although most

382 reviews of postoperative endophthalmitis presume it is due to bacterial infection, even if culture

383 negative (Kamalarajah et al, 2004; Endophthalmitis Study Group, 2007; Haripriya et al, 2018).

384 If postoperative endophthalmitis does occur in the true absence of bacterial infection, none of the

385 proposed prophylactic strategies would be expected to be effective. Similarly, post-

386 phacoemulsification endophthalmitis must be differentiated from toxic anterior segment

387 syndrome (TASS). The two conditions have similar clinical signs, but TASS is marked by

388 peracute onset (usually within 24 hours of surgery), whereas post-phacoemulsification

389 endophthalmitis can be delayed up to 6 weeks or more. Other distinguishing features include the

390 degree of ocular discomfort, flare/cell/hypopyon response, and vision loss, all of which are

391 minimal in TASS cases but severe in endophthalmitis cases (Sengillo et al, 2020).

\section{Conclusions}


393 Our data support the hypothesis that prolonged postoperative antibiotic administration

394 does not demonstrably reduce the rate of post-phacoemulsification endophthalmitis, and we

395 recommend against this practice.

396 Footnotes

397 a SigmaPlot 14, Systat Software, San Jose CA 


\section{References}

399 1. Aaberg TM, Flynn HW, Schiffman J, Newton J. 1998. Nosocomial acute-onset

400 postoperative endophthalmitis survey. A 10-year review of incidence and outcomes.

$401 \quad$ Ophthalmology 105:1004-1010.

402 2. Ang GS, Barras CW. 2006. Prophylaxis against infection in cataract surgery: a survey of 403 routine practice. European Journal of Ophthalmology 16:394-400.

404 3. Azoulay T, Dulaurent T, Isard PF, Poulain N, Goulle F. 2013. Immediately sequential 405 406 bilateral cataract surgery in dogs: A retrospective analysis of 128 cases (256 eyes). Journal Français d'Ophtalmologie 36:645-651 DOI: 10.1016/j.jfo.2012.09.010.

407

408

409

410

411

412

413

414

415

416

417

418

4. Behndig A, Cochener B, Güell JL, Kodjikian L, Mencucci R, Nuijts RMMA, Pleyer U, Rosen P, Szaflik, Tassignon M-J. 2013. Endophthalmitis prophylaxis in cataract surgery: Overview of current practice patterns in 9 European countries. Journal of Cataract and Refractive Surgery 39:1421-1431 DOI: 10.1016/j.jcrs.2013.06.014.

5. Boldy KL. Current status of canine cataract surgery. 1988. Seminars in Veterinary Medicine and Surgery (Small Animal) 3:62-68.

6. Bratzler DW, Houck PM, Richards C, Steele L, Dellinger EP, Fry DE, Wright C, Ma A, Carr K, Red L. 2005. Use of antimicrobial prophylaxis for major surgery: Baseline results from the national surgical infection prevention project. Archives of Surgery 140:174-182.

7. Bratzler DW, Houck PM. 2005. Antimicrobial prophylaxis for surgery: An advisory statement from the National Surgical Infection Prevention Project. American Journal of Surgery 189:395-404 DOI: 10.1016/j.amjsurg.2005.01.015. 
419 8. Cao H, Zhang L, Li L, Lo S. 2013. Risk factors for acute endophthalmitis following cataract

420 surgery: A systematic review and meta-Analysis. PLoS One 8:e71731 DOI:

$421 \quad$ 10.1371/journal.pone.0071731.

422 9. Ciulla TA, Starr MB, Masket S. 2002. Bacterial endophthalmitis prophylaxis for cataract 423 surgery: An evidence-based update. Ophthalmology 109:13-24.

424 10. De Almeida M, Gerrard C, Freeman JT, Duffy E, Roberts SA. 2018. Inappropriate 425 prescribing of antibiotics following discharge after major surgery: an area for improvement. $426 \quad$ New Zealand Medical Journal 131:35-43.

427 11. de Kaspar HM, Chang RT, Shriver EM, Singh K, Egbert PR, Blumenkranz MS, Ta CN. 428 2004. Three-day application of topical ofloxacin reduces the contamination rate of 429 microsurgical knives in cataract surgery: A prospective randomized study. Ophthalmology $430 \quad$ 111:1352-1355 DOI: 10.1016/j.ophtha.2003.10.032.

431 12. Dickey JB, Thompson KD, Jay WM. 1991. Anterior chamber aspirate cultures after 432 uncomplicated cataract surgery. American Journal of Ophthalmology 112:278-282 DOI: 10.1016/S0002-9394(14)76728-5.

434 13. Durand ML. 2013. Endophthalmitis. Clinical Microbiology and Infection. 19:227-234 435 DOI: $10.1111 / 1469-0691.12118$.

436 14. ESCRS Endophthalmitis Study Group, European Society of Cataract \& Refractive Surgeons. 437 2007. Prophylaxis of postoperative endophthalmitis following cataract surgery: Results of 438 the ESCRS multicenter study and identification of risk factors. Journal of Cataract and 439 Refractive Surgery 33:978-988 DOI: 10.1016/j.jcrs.2007.02.032.

440 15. Gift BW, English RV, Nadelstein B, Weigt AK, Gilger BC. 2009. Comparison of capsular 441 opacification and refractive status after placement of three different intraocular lens implants 
442 following phacoemulsification and aspiration of cataracts in dogs. Veterinary

443 Ophthalmology 12:13-21.

444 16. Grzybowski A, Kuklo P, Pieczynski J, Beiko G. A review of preoperative manoeuvres for 445 prophylaxis of endophthalmitis in intraocular surgery: topical application of antibiotics, 446 disinfectants, or both? Current Opinion in Ophthalmology 2016 Jan;27(1):9-23.

447 doi:10.1097/ICU.0000000000000216.

448 17. Haripriya A, Baam Z, Chang DF. 2017. Endophthalmitis prophylaxis for cataract surgery. Asia-Pacific Journal of Ophthalmology 6:324-329 DOI: 10.22608/APO.2017200

18. Haripriya A, Chang DF. 2018. Intracameral antibiotics during cataract surgery: evidence and barriers. Current Opinion in Ophthalmology 29:33-39

19. Hazra S, De D, Roy B, Bose A, Nandi S, Konar A. 2008. Use of ketamine, xylazine, and 453 diazepam anesthesia with retrobulbar block for phacoemulsification in dogs. Veterinary Ophthalmology 11:255-259.

20. Huang J, Wang X, Chen X, Song Q, Liu W, Lu L. 2016. Perioperative antibiotics to prevent acute endophthalmitis after ophthalmic surgery: A systematic review and meta-analysis. PLoS One. Nov 8;11(11):e0166141. doi: 10.1371/journal.pone.0166141. eCollection 2016.

21. Inoue T, Usui N, Kobayakawa S, Ichihara K, Ohashi Y. 2018. Incidence of endophthalmitis and the perioperative practices of cataract surgery in Japan: Japanese prospective multicenter study for postoperative endophthalmitis after cataract surgery. Japanese Journal of Ophthalmology 62:24-30 doi.org/10.1007/s10384-017-0545-6.

22. Johnstone N, Ward DA. 2005. The incidence of posterior capsule disruption during phacoemulsification and associated postoperative complication rates in dogs: 244 eyes (1995-2002). Veterinary Ophthalmology 8:47-50. 
465 23. Kamalarajah S, Silvestri G, Sharma N, Khan A, Foot B, Ling R, Cran G, Best R. 2004.

466 Surveillance of endophthalmitis following cataract surgery in the UK. Eye 2004; 580-587

467 DOI: $10.1038 /$ sj.eye.6700645.

468 24. Kosacki J, Boisset S, Maurin M, Cornut P, Thuret G, Hubanova R, Vandenesch F, Carricajo 469 A, Aptel F, Chiquet C (on behalf of the friends group). 2020. Specific PCR and quantitative 470 real-time PCR in ocular samples from acute and delayed-onset postoperative endophthalmitis. Am J Ophthalmol 212:34-42 doi.org/10.1016/j.ajo.2019.11.026.

25. Lacerda LCC, de Souza-Pollo A, Padua IRM, Conceição LF, da Silveira CPB, Silva GA, Maluta RP, Laus JL. 2018. Molecular characterization and potential sources of aqueous humor bacterial contamination during phacoemulsification with intraocular lens implantation in dogs. Veterinary Microbiology 213:95-101.

26. Landry MP, Herring IP, Panciera DL. 2004. Fundoscopic findings following cataract extraction by means of phacoemulsification in diabetic dogs: 52 cases (1992-2003). Journal of the American Veterinary Medical Association 225:709-716.

27. Ledbetter EC, Millichamp NJ, Dziezyc J. 2004. Microbial contamination of the anterior chamber during cataract phacoemulsification and intraocular lens implantation in dogs. Veterinary Ophthalmology 7:327-334.

28. Ledbetter EC, Spertus CB, Kurtzman RZ. 2018. Incidence and characteristics of acute-onset 483 Association 253:201-208 doi: 10.2460/javma.253.2.201. 
486 487 488 489 490 492 493 494 495 496 497 498

29. Lehmann OJ, Roberts CJ, Ikran K, Campbell MJ, McGill JI. 1997. Association between nonadministration of subconjunctival cefuroxime and postoperative endophthalmitis. Journal of Cataract and Refractive Surgery 23:889-893.

30. Li J, Morlet N, Ng JQ, Semmens JB, Knuiman MW. 2004. Significant nonsurgical risk factors for endophthalmitis after cataract surgery: EPSWA fourth report. Investigative Ophthalmology and Visual Science 45:1321-1328 DOI: 10.1167/iovs.03-1000

31. Liesegang TJ. 1999. Perioperative antibiotic prophylaxis in cataract surgery. Cornea $18: 383-402$.

32. Liesegang TJ. 2001. Use of antimicrobials to prevent postoperative infection in patients with cataracts. Current Opinion in Ophthalmology 12:68-74.

33. Mayer E, Cadman D, Ewings P, Twomey JM, Gray RH, Claridge KG, Hakin KN, Bates AK. 2003. A 10 year retrospective survey of cataract surgery and endophthalmitis in a single eye unit: Injectable lenses lower the incidence of endophthalmitis. British Journal of Ophthalmology 87:867-869 DOI: 10.1136/bjo.87.7.867.

34. Miller JJ, Scott IU, Flynn HW, Smiddy WE, Newton J, Miller D. 2005. Acute-onset endophthalmitis after cataract surgery (2000-2004): Incidence, clinical settings, and visual acuity outcomes after treatment. American Journal of Ophthalmology 139:983-987.

35. Ou JI, Ta CN. 2006. Endophthalmitis prophylaxis. Ophthalmology Clinics of North America 19:449-456 DOI: 10.1016/j.ohc.2006.07.005.

36. Paulsen ME, Lavach JD, Severin GA, Eichenbaum JD. 1986. The effect of lens-induced uveitis on the success of extracapsular cataract extraction: A retrospective study of 65 lens removals in the dog. Journal of the American Animal Hospital Association 22:49-56.

Peer] reviewing PDF | (2021:05:60898:1:1:NEW 26 Aug 2021) 
508 37. Rahmani S, Eliott D. 2018. Postoperative endophthalmitis: a review of risk factors, 509 prophylaxis, incidence, microbiology, treatment, and outcomes. Seminars in Ophthalmology

$510 \quad 33: 95-101$ DOI: $10.1080 / 08820538.2017 .1353826$.

511 38. Rosha DS, Ng JQ, Morlet N, Boekelaar M, Wilson S, Hendrie D, Semmens JB. 2006.

512 Cataract surgery practice and endophthalmitis prevention by Australian and New Zealand

513 ophthalmologists. Clinical and Experimental Ophthalmology 34:535-544 DOI: 10.1111/j.1442-9071.2006.1276.x

39. Rudnisky CJ, Wan D, Weis E. 2014. Antibiotic choice for the prophylaxis of post-cataract 516 extraction endophthalmitis. Ophthalmology 121:835-841 DOI: 10.1111/j.1442$517 \quad 9071.2006 .1276 . x$.

518 40. Schmitz S, Dick HB, Krummenauer F, Pfeiffer N. 1999. Endophthalmitis in cataract 519 surgery: Results of a German survey. Ophthalmology 106:1869-1877.

520 41. Sengillo JD, Chen Y, Garcia DP, Schwartz SG, Grzybowski A, Flynn HW. 2020.

521 Postoperative endophthalmitis and toxic anterior segment syndrome prophylaxis: 2020 update. Annals of Translational Medicine 8:1548-1560 doi.org/10.21037/atm-2019-rcs-02

42. Sherwood DR, Rich WJ, Jacob JS, Hart RJ, Fairchild YL. 1989. Bacterial contamination of 524 intraocular and extraocular fluids during extracapsular cataract extraction. Eye 3:308-312.

43. Sigle KJ, Nasisse MP. 2006. Long-term complications after phacoemulsification for cataract removal in dogs: 172 cases (1995-2002). Journal of the American Veterinary Medical Association 228:74-79.

528 44. Speaker MG, Menikoff JA. 1991. Prophylaxis of endophthalmitis with topical povidone529 iodine. Ophthalmology 98:1769-1775. 
530 45. Ta CN, Egbert PR, Singh K, Shriver EM, Blumenkranz MS, de Kaspar HM. 2002.

531 Prospective randomized comparison of 3-day versus 1-hour preoperative ofloxacin

532 prophylaxis for cataract surgery. Ophthalmology 109:2036-2041.

533 46. Taban M, Behrens A, Newcomb RL, Nobe MY, Saedi G, Sweet PM, McDonnell PJ. 2005.

534 Acute endophthalmitis following cataract surgery. A systematic review of the literature.

535 Archives of Ophthalmology 123:613-620.

536 47. Taylor MM, Kern TJ, Riis RC, McDonough PL, Erb HN. 1995. Intraocular bacterial

537 contamination during cataract surgery in dogs. Journal of the American Veterinary Medical

538 Association 206:1716-1720.

539 48. Tervo T, Ljungberg P, Kautiainen T, Puska P, Lehto I, Raivio I, Järvinen E, Kuusela P,

540 Tarkkanen A. 1999. Prospective evaluation of external ocular microbial growth and

541 aqueous humor contamination during cataract surgery. Journal of Cataract and Refractive

$542 \quad$ Surgery 25:65-71.

543 49. Therese KL, Anand AR, Madhavan HN. 1998. Polymerase chain reaction in the diagnosis

544 of bacterial endophthalmitis. British Journal of Ophthalmology 82:1078-1082

545 doi: $10.1136 /$ bjo.82.9.1078.

546 50. Weese JS, Giguère S, Guardabassi L, Morley PS, Papich M, Ricciuto DR, Sykes JE. 2015.

547 ACVIM consensus statement on therapeutic antimicrobial use in animals and antimicrobial

548 resistance. Journal of Veterinary Internal Medicine 29:487-498 DOI: 10.1111/jvim.12562.

549 51. Wejde G, Samolov B, Seregard S, Koranyi G, Montan PG. 2005. Risk factors for

550 endophthalmitis following cataract surgery: A retrospective case-control study. Journal of

$551 \quad$ Hospital Infection 61:251-256 DOI: 10.1016/j.jhin.2005.04.016 


\section{Table $\mathbf{1}$ (on next page)}

Table 1. Comparison of group receiving postoperative oral antibiotics with group not receiving postoperative oral antibiotics. 
2 Table 1. Comparison of group receiving postoperative oral antibiotics with group not receiving

3 postoperative oral antibiotics.

\begin{tabular}{|c|c|c|c|}
\hline & $\begin{array}{c}\text { Group receiving } \\
\text { postoperative oral } \\
\text { antibiotics } \\
\end{array}$ & $\begin{array}{c}\text { Group NOT receiving } \\
\text { postoperative oral } \\
\text { antibiotics } \\
\end{array}$ & $p$ \\
\hline No. of patients & 112 & 103 & \\
\hline No. of eyes & 197 & 171 & \\
\hline $\begin{array}{l}\text { No. of eyes diagnosed with } \\
\text { endophthalmitis }\end{array}$ & 1 & 2 & 0.57 \\
\hline \multicolumn{4}{|l|}{ Breeds $^{1}$} \\
\hline Mixed breed dog & $20(18 \%)$ & $16(16 \%)$ & \\
\hline Boston Terrier & $14(13 \%)$ & $15(15 \%)$ & \\
\hline Cocker Spaniel & $14(13 \%)$ & $8(8 \%)$ & \\
\hline Miniature Poodle & $11(10 \%)$ & $6(6 \%)$ & \\
\hline Bichon Frise & $7(6 \%)$ & $6(6 \%)$ & \\
\hline Miniature Schnauzer & $7(6 \%)$ & $6(6 \%)$ & \\
\hline Toy Poodle & $7(6 \%)$ & $7(7 \%)$ & \\
\hline Age (median (25\%, 75\%); yrs) & $7(4,9)$ & $7(4,10)$ & 0.73 \\
\hline \multicolumn{4}{|l|}{ No. eyes with IOLs } \\
\hline $\begin{array}{l}\text { PMMA (n (\% of eyes } \\
\text { w/known IOL status)) }\end{array}$ & $109(64 \%)$ & $117(68 \%)$ & \multirow[t]{3}{*}{0.09} \\
\hline Acrylic & $18(11 \%)$ & $26(15 \%)$ & \\
\hline Aphakic & $43(25 \%)$ & $28(16 \%)$ & \\
\hline Not recorded & 27 & 0 & \\
\hline $\begin{array}{l}\text { No. eyes operated by } \\
\text { faculty/residents }\end{array}$ & $92 / 105(47 \% / 53 \%)$ & $83 / 88(49 \% / 51 \%)$ & 0.26 \\
\hline No. diabetic patients & $18(16 \%)$ & $23(22 \%)$ & 0.18 \\
\hline $\begin{array}{l}\text { No. eyes with IOP }>25 \mathrm{mmHg} \\
\text { within } 24 \text { hours of surgery }\end{array}$ & $46(23 \%)$ & $35(20 \%)$ & 0.27 \\
\hline $\begin{array}{l}\text { Operative time/eye (median } \\
\quad(25 \%, 75 \%) \text {; mins })\end{array}$ & $62(51,70)$ & $63(50,75)$ & 0.98 \\
\hline Phacoemulsification machine ${ }^{2}$ & AMO Diplomax $(n=197)$ & $\begin{array}{l}\text { AMO Diplomax }(n=152) \\
\text { Alcon Infiniti }(n=19)\end{array}$ & \\
\hline
\end{tabular}

4

5120 non-listed breeds in the antibiotic-treated group and 22 non-listed breeds in the non-antibiotic-

6 treated group had fewer than 6 individuals each

$7 \quad{ }^{2}$ Both phacoemulsification machines used in this study utilize peristaltic pump technology. All

8 endophthalmitis cases were operated using the AMO Diplomax unit. 